\title{
ZGMTH $\begin{gathered}\text { Zeitschift der } \\ \text { Cesellschat tir Musikheorie }\end{gathered}$
}

Sprick, Jan Philipp (2011): Editorial. ZGMTH 8/3, 387-388.

https://doi.org/10.31751/653

(C) 2011 Jan Philipp Sprick

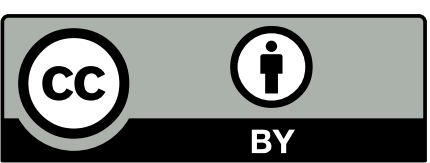

Dieser Text erscheint im Open Access und ist lizenziert unter einer Creative Commons Namensnennung 4.0 International Lizenz.

This is an open access article licensed under a

Creative Commons Attribution 4.0 International License.

veröffentlicht / first published: 29/03/2012

zuletzt geändert / last updated: 09/11/2013 


\section{Editorial}

Die in dieser Varia-Ausgabe der ZGMTH versammelten Beiträge widmen sich in erster Linie Fragen der Satztechnik und der musikalischen Analyse. Vielfach durchdringen sich hierbei historische, systematische und empirische Ansätze und führen auf diese Weise zu einem Methodenpluralismus, der charakteristisch für die gegenwärtige Physiognomie der deutschsprachigen Musiktheorie sein dürfte. Zudem verdankt die Mehrzahl der Beiträge ihr Thema bzw. ihre Fragestellung dem Dialog mit der pädagogischen Praxis, was eine Bestimmung des Verhältnisses von Wissenschaft und Lehre mit einschließt, ohne dass dieses Verhältnis in der Regel explizit zum Thema gemacht würde. So können die jeweiligen Arbeitsergebnisse für sich stehen und vermögen doch zugleich Anregungen für die konkrete Unterrichtspraxis zu geben. 'Wissenschaftliche ‘ Musiktheorie und pädagogische Praxis erscheinen vor diesem Hintergrund nicht als unabhängige, sondern miteinander verwobene Bereiche.

Johannes Menke zeichnet in seinem Beitrag die Geschichte der cadenza doppia nach. Beginnend mit den ersten Quellen im 16. Jahrhundert führt seine Beschreibung von der Entfaltung des kadenziellen und sequenziellen Potenzials der Formel im 17. und 18. Jahrhundert bis hin zu ihrer bisweilen archaisch anmutenden Verwendung in der Musik des 19. Jahrhunderts.

Einem in der deutschsprachigen Musiktheorie nur selten thematisierten Werkkorpus widmet sich Tihomir Popovic in seinem Beitrag zur »Tonartbehandlung in William Byrds Fantasien für Tasteninstrumente«. Die musiktheoretischen Quellen stehen hier im Dienste einer kontextualisierenden Perspektive, die die Ergebnisse der musikalischen Analyse auch sozial- und kulturgeschichtlich interpretiert.

Hinsichtlich des Werkkorpus' verhält es sich bei der empirischen Untersuchung von Andreas Moraitis zu den »Stimmführungsparallelen in den Choralsätzen Johann Sebastian Bachs und Georg Philipp Telemanns" genau umgekehrt: Es gibt wahrscheinlich kein stärker kanonisiertes `musiktheoretisches Repertoire` als die vierstimmigen Choralsätze der ersten Hälfte des 18. Jahrhunderts. Obwohl die in Bezug auf dieses Repertoire häufig diskutierte Frage nach den Stimmführungsparallelen in nahezu jedem Tonsatzlehrgang thematisiert wird, kann Moraitis auf der Basis einer computergestützten Auswertung von annähernd 1000 Sätzen zu einer weiteren Ausdifferenzierung der für dieses Phänomen relevanten Satzregeln beitragen.

Aus einem kritischen Impuls gegenüber etablierten Verfahren entwickeln die beiden Beiträge in der Rubrik »Musiktheorie der Gegenwart« neue Analyseansätze für zwei zentrale musikalische Parameter: Ludwig Holtmeier stellt in seinem auf zwei Teile angelegten Text »Funktionale Mehrdeutigkeit, Tonalität und arabische Stufen« die Frage, "welchen analytischen Zeichensystems sich eine historisch informierte Satzlehre bedienen« solle. Sein Vorschlag zu einer »Reform der harmonischen Analyse« ist im Kern eine Weiterentwicklung von Emanuel Aloys Försters arabischer Stufenanalyse zu Beginn des 
19. Jahrhunderts, die insbesondere der «funktionalen Mehrdeutigkeit« einzelner Akkorde gerecht werden möchte. Peter Petersen stellt in seinem Beitrag »Primäre und sekundäre Dauern in der Musik« eine «omponententheorie` zur Diskussion, die in der Rhythmusanalyse auch die Dauern zwischen »zwischen Tönen/Klängen sowie Klanggestalten und deren Komponenten « zu berücksichtigen sucht, um so ein differenzierteres Verständnis rhythmischer Prozesse zu erreichen.

Den Abschluss machen zwei Rezensionen und ein Bericht: Skepsis gegenüber dem »universellen Geltungsanspruch musiktheoretischer Systeme« spricht aus Felix Stephans Doppelrezension von Reinhard Amons Lexikon der Harmonielehre und Lexikon der musikalischen Form. Zwar gebe es »derzeit keine anderen deutschsprachigen Publikationen«, so Stephan, die »ein so breites Überblickswissen in vergleichbar gebündelter und vertiefter Form verfügbar machen«, doch lasse Amon bisweilen jenen Grad historischer Differenzierung vermissen, der im Fachdiskurs mittlerweile Standard sei.

Ulrich Kaiser bespricht Dietmar Elfleins Studie Schwermetallanalysen, die sich innerhalb des in der deutschsprachigen Musiktheorie ohnehin eher stiefmütterlich behandelten Bereichs der Popularmusik durch einen ungewöhnlichen analytischen Schwerpunkt auszeichnet. Elfleins Buch, so Kaiser, leiste, indem es zu »konkreten Aussagen über die Musik des Heavy Metal« gelange, einen "gewichtigen deutschsprachigen Beitrag zur Erforschung der Populären Musik».

Mein Bericht vom Jahrestreffen der Society for Music Theory in Minneapolis im November 2011 spiegelt eine Tendenz zu interdisziplinären Fragestellungen in der nordamerikanischen Musiktheorie, die »mittelfristig nicht nur die Music Theory selbst, sondern auch das Verhältnis zu den beiden Nachbardisziplinen Musicology und Ethnomusicology nachhaltig verändern« dürfte, zugleich aber den wissenschaftlichen Diskurs zunehmend von der Unterrichtsrealität des Faches entkoppelt.

Vor diesem Hintergrund scheint gerade die Verbindung von wissenschaftlicher Reflexion und pädagogischer Praxis in der deutschsprachigen Musiktheorie nicht mehr bloß die institutionelle Situation des Faches abzubilden, sondern vielmehr zu einem eigenständigen inhaltlichen Profil zu führen.

Jan Philipp Sprick 